\title{
MAGNETIC POLARON FORMATION IN SEMIMAGNETIC SEMICONDUCTOR HETEROSTRUCTURES
}

\author{
D.R. YAKOVLEV \\ Physikalisches Institut der Universität Würzburg, 97074 Würzburg, Germany \\ and \\ A.F. Ioffe Physico-Technical Institute, Russian Academy of Sciences \\ 194021 St. Petersburg, Russia
}

We present a survey of experimental and theoretical studies of exciton magnetic polarons in semimagnetic semiconductor epilayers, quantum wells and superlattices. The emphasis is on dynamical aspects of magnetic polaron formation in systems of different dimensionality.

PACS numbers: $78.20 . \mathrm{Ls}, 71.35 .+\mathrm{z}, 75.50 . \mathrm{Rr}$

\section{Introduction}

In semimagnetic semiconductors, carriers are coupled with localized spins of magnetic ions by a strong exchange interaction [1]. This interaction causes the ferromagnetic alignment of the localized spins in the vicinity of the carrier. The resulted cloud of polarized spins can be considered as a magnetic molecule with magnetic moment of hundreds Bohr magnetons. Spin-organized systems of this kind are called magnetic polarons (MPs). Theoretical studies of the magnetic polarons have been pioneered by De Gennes [2]. Existence of MPs in the magnetic semiconductors like EuO was established via transport and magnetization measurements [3]. But detailed information about the energy spectrum and spin structure of MPs was not available from these experiments. The situation has been changed after appearance of the semimagnetic semiconductors, where powerful optical methods can be applied for a study of MP states. (Cd,Mn)Te and $(\mathrm{Cd}, \mathrm{Mn}) \mathrm{Se}$, where the magnetic properties are controlled by a concentration of magnetic Mn ions, were widely used for these studies. First effort of the theory, inspired by Raman and photoluminescence studies of MPs bound to acceptors or donors, was aimed at the description of the internal spin structure of a bound MP (BMP) with a fixed localization radius. The coordinate part of the carrier wave function in BMPs is prescribed by the Coulomb potential of an impurity center, and the carrier lifetime on the center is usually much longer than the characteristic times of spin relaxation in the MP. Now the main features of BMPs are understood 
fairly well (for review see Ref. [4]). With experimental findings on exciton magnetic polarons (EMPs) [5-9] there appeared a series of new questions connected with localization conditions of excitons and dynamics of spin organization in the polaron formation process. These specific features distinguish the exciton magnetic polarons from the BMPs:

(i) Localization area and, respectively, the energy of EMP ground state are not determined by the Coulomb potential of the impurity center, but depend on the exciton localization or self-localization conditions. Moreover, the exciton autolocalization can play very important role, contributing to the MP energy and affecting the MP dynamics.

(ii) Exciton lifetime is limited by recombination processes (both radiative and nonradiative). In II--VI semiconductors it is comparable with the EMP formation time. As a result, the process of the magnetic polaron formation can be interrupted by exciton recombination before the MP reaches its equilibrium energy. The dynamics of MP formation itself depends on exciton localization conditions, temperature and external magnetic fields. It also depends strongly on the concentrations of magnetic Mn ions. On the one hand, the exchange energy gained in the process of the MP formation is determined by the Mn concentration (this dependence is nonlinear and nonmonotonic because of antiferromagnetic coupling of nearest-neighbor Mn spins [1]), and on the other hand a system of interacting Mn spins plays an important role for energy and spin dissipation in the process of MP formation $[10,11]$.

Recent progress in the semiconductor growth technology, mainly due to molecular-beam epitaxy, allows to create high quality structures with quantum wells and superlattices containing thin layers of semimagnetic semiconductors. It made possible to extend the studies of the EMP formation into the field of low-dimensional systems $[12,13]$. Theoretical estimations predict that the stability conditions for free magnetic polarons (FMPs) (i.e. self-localized MPs) are hardly realized in the bulk semimagnetic semiconductors, but they are favored strongly by the reduction of dimensionality and the FMP is always stable in the one-dimensional system $[14,15]$.

In this paper we give a survey of experimental and theoretical studies of the exciton magnetic polaron formation in three-dimensional (3D) and two-dimensional (2D) systems realized in semimagnetic semiconductor epilayers, quantum well (QW) structures and superlattices (SLs). We should focus our attention on the dynamical aspects of the magnetic polaron formation.

\section{Theoretical aspects}

The problem of the stability of free magnetic polarons was first analyzed by Kasuya et al. for 3D systems [16] and by Ryabchenko and Semenov for low-dimensional systems [17]. On appearance of semimagnetic QWs, an analysis of exciton FMPs in these structures was attempted by A. Kavokin and K. Kavokin [15, 18] and by Benoit à la Guillaume [14]. It has been shown that the FMP formation is favored by the reduction of dimensionality and by the increase in exchange constants and carrier effective masses. It follows from results of model calculations for the semimagnetic semiconductors $[14,19]$ that: 
(i) Self-localization in 3D systems is very unfavorable and the initial localization of excitons is of key importance for MP formation. We label such MPs formed from localized excitons as localized MPs (LMPs) [20]. The radius of the exciton localization is not expected to decrease significantly in the process of the LMP formation.

(ii) In experimentally available 2D systems initial localization is still necessary to start the MP formation. On the other hand, the localization radius can change several times during the polaron formation. MPs of this kind can be considered as quasi-free MPs.

Simple considerations given below show that the exciton autolocalization, which is accompanied by a shrinkage of the exciton wave function, can affect not only the equilibrium energy of the EMP, but also the dynamics of its formation [21]. We consider the process of MP formation from exciton with some degree of initial localization. Let the evolution of the magnetization in the Mn spin system $M(r, t)$ obey the simplest first-order differential equation with the unique relaxation time $\tau_{\mathrm{s}}$ :

$$
\frac{\mathrm{d}}{\mathrm{d} t} M(r, t)=-\frac{1}{\tau_{\mathrm{s}}}\left[M(r, t)-M_{\mathrm{eq}}\left(B_{\mathrm{ex}}(r, t)\right)\right]
$$

where $M_{\text {eq }}$ is the equilibrium magnetization which corresponds to the exchange field $B_{\text {ex }}(r, t)=\alpha|\Psi(r, t)|^{2}$ created by the exciton, here $\alpha$ is the exchange constant. By averaging Eq. (1) with the exciton wave function $\Psi(r, t)$, and making use of the well-known result of the perturbation theory for the first-order correction to energy in respect of a change of the magnetization: $\delta \varepsilon=\alpha\langle\delta M\rangle$, one can derive the following equation for the polaron energy $\varepsilon(t)=E(t)+\alpha\langle M(t)\rangle$ :

$$
\frac{\mathrm{d}}{\mathrm{d} t} \varepsilon(t)=-\frac{1}{\tau_{\mathrm{s}}}\left[\varepsilon(t)-\varepsilon_{\mathrm{eq}}(t)\right]
$$

where $\varepsilon_{\mathrm{eq}}(t)=E(t)+\alpha\left\langle M_{\mathrm{eq}}(t)\right\rangle, E(t)$ is a non-magnetic part of the energy. Inasmuch as the energy $\varepsilon$ is a monotonic function of time, $\varepsilon_{\text {eq }}$ can be considered as a function of $\varepsilon$. With knowledge of the dependence $\varepsilon_{\mathrm{eq}}(\varepsilon)$, the function $\varepsilon(t)$ which describes the dynamics of the MP formation can be obtained from Eq. (2).

To estimate the characteristic time of the MP formation, let us summarize our knowledge about the dependence $\varepsilon_{\text {eq }}(\varepsilon)$ for localized MPs. In the initial state, where $\varepsilon=0$, $\varepsilon_{\text {eq }}$ has a finite value $\varepsilon_{\text {eq }}(0)=\alpha\left\langle M_{\text {eq }}\left(B_{\text {ex }}(0)\right)\right\rangle$ which is determined by the conditions of initial localization. When the polaron reaches its final state with the energy $\varepsilon_{\mathfrak{f}}=\varepsilon(t \rightarrow \infty)$, the polaron energy reaches its equilibrium value: $\varepsilon_{\text {eq }}\left(\varepsilon_{\mathfrak{f}}\right)=\varepsilon_{\mathfrak{f}}$.

For intermediate states we approximate $\varepsilon_{\text {eq }}(\varepsilon)$ with the use of a linear interpolation

$$
\varepsilon_{\mathrm{eq}}(\varepsilon)=\varepsilon_{\mathrm{eq}}(0)+\left(\varepsilon_{\mathrm{f}}-\varepsilon_{\mathrm{eq}}(0)\right) \frac{\varepsilon}{\varepsilon_{\mathrm{f}}} .
$$

Substitution of this dependence into Eq. (2) results in an exponential law of relaxation

$$
\varepsilon(t)=\varepsilon_{\mathrm{f}}\left(1-\mathrm{e}^{-t / \tau_{\mathrm{i}}}\right)
$$

where the formation time

$$
\tau_{\mathrm{f}}=\tau_{\mathrm{s}} \frac{\varepsilon_{\mathrm{f}}}{\varepsilon_{\mathrm{eq}}(0)}=\tau_{\mathrm{s}}(1+\xi) \text {. }
$$


$\xi>0$ is the fraction of the polaron energy gained through autolocalization. For all studied structures the experimentally measured dynamics of the MP formation is very well described by the exponential law, which in turn confirms the validity of the chosen approximation for $\varepsilon_{\text {eq }}(\varepsilon)$.

Analyzing Eq. (5), one can conclude that in 3D systems, where the autolocalization of MP is negligible, $\tau_{\mathfrak{f}}=\tau_{\mathrm{s}}$, i.e. polaron formation is determined by the relaxation dynamics in the system of $\mathrm{Mn}$ ions only. In QWs, where the autolocalization contributes considerably the MP equilibrium energy, $\tau_{\mathrm{f}}$ exceeds $\tau_{\mathrm{s}}$ by the factor determined in Eq. (5). It is worthwhile to note that the above analysis cannot be applied to the free magnetic polarons, where $\varepsilon_{\text {eq }}(0)=0$ and $\mathrm{Eq}$. (5) gives an infinite formation time. In this case Eq. (3) should be refined by an account for nonlinear terms in $\varepsilon_{\text {eq }}(\varepsilon)$.

In the following, we discuss first the autolocalization contribution in the dynamics of MP formation and then consider the relaxation processes in the Mn spin system, which determine $\tau_{\mathrm{s}}$.

\section{Experimental results and discussion}

Methods of optical spectroscopy, namely photoluminescence (PL) and polarized PL, allow to get detailed information about static and dynamic properties of MPs in semimagnetic semiconductors. MP formation causes exciton localization in the exchange potential and the polaron energy can be determined from a low energy shift of a luminescence line in respect of the free exciton energy. In the semimagnetic semiconductors the Stokes shift of PL line is due to both polaron formation and exciton localization in nonmagnetic random potential, and it is necessary to distinguish between these two contributions. We use a method of selective excitation for the EMP studies in $\mathrm{Cd}_{1-x} \mathrm{Mn}_{x}$ Te-based epilayers and heterostructures grown by molecular-beam epitaxy [20,22]. Excitons are excited selectively in the band of localized states at energies where the spectral diffusion due to phonon-assisted tunneling does not occur during the exciton lifetime. In this case the Stokes shift $\Delta E$ between the luminescence line and the energy of selective excitation is determined by the magnetic polaron formation only.

The dynamics of MP formation has been studied by a picosecond time-resolved spectroscopy $[20,23]$. The PL signal was recorded by a streak-camera with temporary resolution of $15 \mathrm{ps}$ and selective photoexcitation was provided by $5 \mathrm{ps}$ laser pulses. An inset in Fig. 1 shows the time evolution of the magnetic polaron shift $\Delta E$ in three $\mathrm{Cd}_{1-x-y} \mathrm{Mn}_{x} \mathrm{Mg}_{y}$ Te epilayers at $1.6 \mathrm{~K}$. The fast initial shift of the polaron line is followed by a saturation of $\Delta E(t)$ at the level of $\Delta E(t \rightarrow \infty)$, which corresponds to the equilibrium energy of MP $\left(E_{\mathrm{MP}}\right)$. The formation process is well described by an exponential law $\Delta E(t)=\Delta E(t \rightarrow \infty)\left[1-\exp \left(-t / \tau_{\mathfrak{f}}\right)\right]$ for all structures reported in this paper. The polaron lifetimes $\tau_{0}$ have been determined from the decay of the spectrally integrated PL intensity.

Let us first discuss the dynamics of MP formation in 3D and 2D systems without going into details of microscopic mechanisms determining $\tau_{\mathrm{s}}$. The MP formation time as a function of the Mn content in $\mathrm{Cd}_{1-x} \mathrm{Mn}_{x} \mathrm{Te}$ (3D system) is shown in Fig. 1. It decreases from 130 ps down to 50 ps with $x$ increase in the range $0.12-0.33$. The increase in Mn content causes change of the magnetic properties 


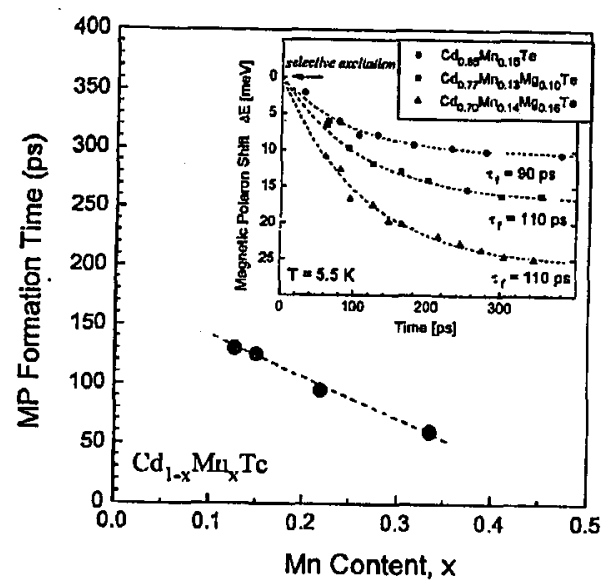

Fig. 1. Magnetic polaron formation time as a function of $\mathrm{Mn}$ content in $\mathrm{Cd}_{1-x} \mathrm{Mn}_{x} \mathrm{Te}$ epilayers [20]. The inset displays time evolution of the MP shift for different quaternary alloys [24]. The dashed lines are exponential fits with $\tau_{\mathrm{f}}$ given in the inset.

and increase in compositional disorder, which in turn leads to a stronger initial localization of excitons and larger MP energies. In order to distinguish between these two factors contributing the polaron formation time, the MP formation was measured in the quaternary alloys $\mathrm{Cd}_{1-x-y} \mathrm{Mn}_{x} \mathrm{Mg}_{y} \mathrm{Te}$ with a nearly identical $\mathrm{Mn}$ content of about 0.14 (see inset of Fig. 1). Despite the considerable increase in the polaron equilibrium energy with increasing $\mathrm{Mg}$ content, the formation times differ only slightly [24]. Therefore, we conclude that the formation time does not scale with the MP energy and with a degree of the initial localization, but is determined by the relaxation dynamics in the system of Mn ions. Remember that in 3D systems the autolocalization of MPs is expected to be negligible and experimentally determined formation time $\tau_{\mathrm{f}}$ can be directly associated with the relaxation time $\tau_{\mathbf{s}}$, i.e. $\tau_{\mathfrak{f}}=\tau_{\mathbf{s}}$ (see Eq. (5)). Therefore, the data in Fig. 1 can be considered as a functional dependence of $\tau_{\mathbf{s}}$ on the Mn content.

A theoretical analysis of the MP dynamics in $2 \mathrm{D}$ systems predicts a considerable contribution of the autolocalization, which is accounted by the factor $\xi$ in Eq. (5): $\tau_{\mathrm{f}}=\tau_{\mathbf{s}}(1+\xi)$. Experimental data from Fig. 2 a for $\mathrm{Cd}_{0.93} \mathrm{Mn}_{0.07} \mathrm{Te} /$ $\mathrm{Cd}_{0.67} \mathrm{Mn}_{0.07} \mathrm{Mg}_{0.26} \mathrm{Te} \mathrm{QWs}$ confirm this expectation [25]. Despite the uniform $\mathrm{Mn}$ content throughout the entire structure, the polaron formation time decreases from 185 ps down to $135 \mathrm{ps}$ by the well width decrease. The well width decrease down from $80 \AA$ causes the enhancement of in-plane exciton localization on the well width fluctuations. That, in turn, reduces the possibility for the autolocalization and shifts $\tau_{\mathbf{f}}$ closer to $\tau_{\mathbf{s}}$. MP lifetimes are displayed in Fig. 2a by open circles. Contrary to the $\tau_{\mathrm{f}}$ behavior, $\tau_{0}$ increases in narrower QWs and in the $20 \AA$ thick QW exceeds $\tau_{\mathrm{f}}$. The smaller is the ratio $\tau_{\mathrm{f}} / \tau_{0}$, the greater polaron shift can be attained and the smaller is the difference between the MP shift detected under $\mathrm{cw}$ conditions and the equilibrium MP energy. For the studied structure both of these energies are plotted in Fig. 2b. 


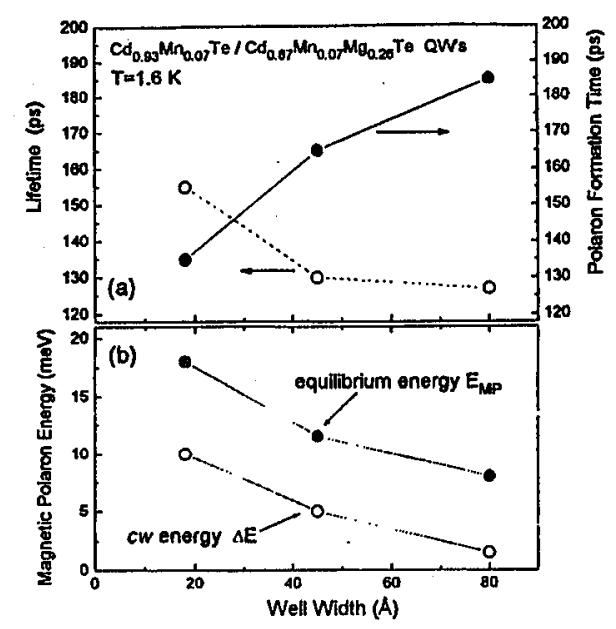

Fig. 2. (a) Polaron formation time (closed circles) and lifetime (open circles) versus well width in $\mathrm{Cd}_{0.93} \mathrm{Mn}_{0.07} \mathrm{Te} / \mathrm{Cd}_{0.67} \mathrm{Mn}_{0.07} \mathrm{Mg}_{0.26} \mathrm{Te}$ QWs; (b) Well width dependence of the MP shifts measured under cw excitation (open circles) and equilibrium MP energies determined under pulsed excitation (closed circles) [25].

Now we are going to discuss relaxation processes which determine the MP formation. The magnetic polaron formation is related to the $\mathrm{Mn}$ spin orientation along the direction of the carrier-induced exchange field $\boldsymbol{B}_{\mathrm{ex}}$. But at the very first time moments after exciton photogeneration the direction of $\boldsymbol{B}_{\text {ex }}$ needs to be stabilized. A directional stabilization of the exciton spin (and respectively, $\boldsymbol{B}_{\text {ex }}$ ) takes place after achievement of the "starting" correlation between exciton and the system of Mn spins, when the MP (exchange) energy exceeds $k_{\mathrm{B}} T$. After stabilization of the $\boldsymbol{B}_{\mathrm{ex}}$ direction, further evolution of the MP state is determined by rates of both energy- and spin relaxation of the Mn spins. The time $\tau_{\mathrm{s}}$ is controlled by the slowest process among energy- and spin relaxation of the $\mathrm{Mn}$ spins.

The polaron formation times shown in Fig. 1 for the 3D systems, where the autolocalization contribution to $\tau_{\mathrm{f}}$ can be neglected and $\tau_{\mathrm{f}}=\tau_{\mathrm{s}}$, indicate that $\tau_{\mathrm{s}}$ varies between 50 and 150 ps and decreases monotonically for higher $\mathrm{Mn}$ contents. A collection of relaxation times for the Mn spins in the semimagnetic semiconductors done by Dietl et al. [10] is displayed in Fig. 3. The spin-lattice relaxation process, which assists energy transfer from the $\mathrm{Mn}$ spins to the phonon system, requires time in the range from $100 \mathrm{~ns}$ to $100 \mu \mathrm{s}$. It is by several orders of magnitude longer than the spin-spin relaxation dynamics of the Mn-spin system which occupies the range from 1 ps to $1 \mathrm{~ns}$. Both of these relaxation processes are accelerated considerably for a $\mathrm{Mn}$ concentration increase, which is accompanied by an enhancement of the spin-spin interactions in the Mn-spin system. The experimentally determined MP formation times for $x=0.1-0.35$ are shown by arrows in Fig. 3. They correspond remarkably well to the spin-spin relaxation dynamics. Hence, we can exclude the direct coupling between the Mn spins and the phonons 


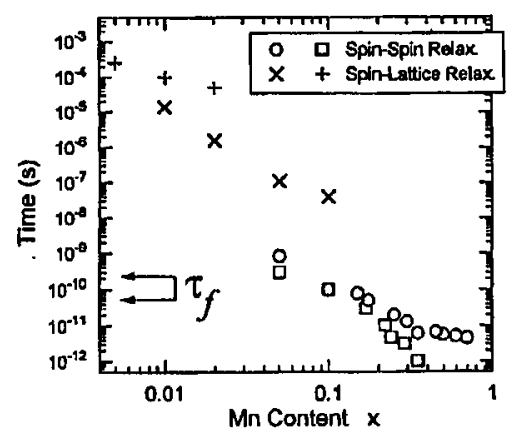

Fig. 3. A collection of spin-lattice and spin-spin relaxation times in $\mathrm{Cd}_{1-x} \mathrm{Mn}_{x} \mathrm{Te}$ at $5 \mathrm{~K}$. Arrows show the range of the magnetic polaron formation time.

from mechanisms which provide the energy relaxation inside short time of MP formation.

Two mechanisms can be suggested for the energy relaxation in MPs:

(i) the excitons themselves being effectively coupled both with the Mn spins and the phonons can provide high spin-lattice relaxation rate for the Mn ions [26];

(ii) the system of interracting Mn spins has an energy reservoir of sufficient capacity, and energy transfer from the Mn spins to this reservoir happens with spin-spin relaxation rates [10]. Changes of the local magnetization inside MP volume require relaxation of the spin momentum. The analysis of the spin diffusion model suggested in Ref. [26] gives a power-law for the dynamics of the MP formation [10]. Thus, the diffusion model is in conflict with the experimental findings, which show the exponential dependence $\Delta E(t)$. It has been suggested recently that the conservation of the spin momentum can be broken by strong nonscalar spin-spin interactions in the Mn system [10]. This process is responsible for a generation of a nonzero magnetization inside the polaron volume.

Figure 4 shows the time evolution of the magnetic polaron shift taken at different repetition rates of excitation pulses [27]. It is clearly seen that the polaron formation time is shortened from 150 ps at $13 \mathrm{~ns}$ pulse separation ( $76 \mathrm{MHz}$ ) down to $90 \mathrm{ps}$ at $117 \mathrm{~ns}$ pulse separation $(8.5 \mathrm{MHz})$. In both cases the equilibrium polaron energy $\Delta E(t \rightarrow \infty)=17 \mathrm{meV}$ and polaron lifetime $\tau_{0}=300 \mathrm{ps}$ remain constant. Therefore, the observed changes in the formation times cannot be related to the heating of paramagnetic Mn spins contributing to the MP formation. We explain this effect in terms of saturation of relaxation channels in the Mn spin system. It follows from our experiment that a recovering time of these relaxation channels is about $100 \mathrm{~ns}$, which is characteristic of the spin-lattice relaxation processes.

The starting correlation stage of MP formation is determined by magnetic fluctuations, which are specific for the semimagnetic semiconductors $[4,28]$. The starting correlation is established when the spin of exciton and the magnetic moment of fluctuation $\left(M_{\mathrm{f}}\right)$ are aligned parallel to each other. A typical exchange energy gained by the exciton in the magnetic fluctuation is about $1 \mathrm{meV}$, which at liquid helium temperatures exceeds strongly $k_{\mathrm{B}} T \approx 0.1 \mathrm{meV}[28,29]$. This means 


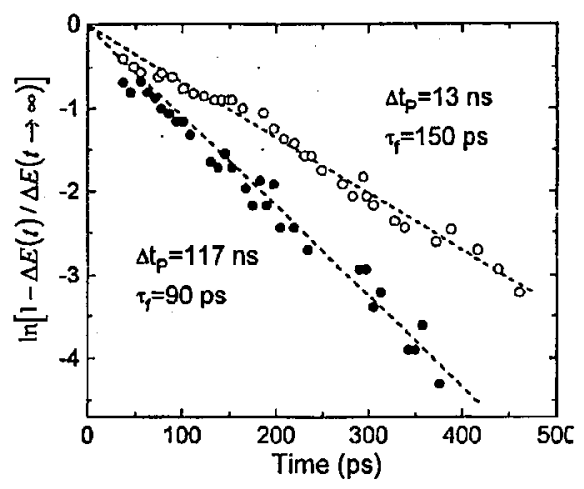

Fig. 4. Time evolution of the polaron shift at two different pulse separations (logarithmic scale) in a $75 \AA$ thick $\mathrm{Cd}_{0.90} \mathrm{Mn}_{0.10} \mathrm{Te} / \mathrm{Cd}_{0.60} \mathrm{Mg}_{0.40} \mathrm{Te}$ quantum well. In this experiment the excitation intensity per pulse is constant [27].

that the energy criterion for the directional stabilization of the exciton spin is satisfied by the magnetic fluctuations. It follows from a comparison of our experimental data with results of model calculation reported below, that the relaxation of the exciton spin on the direction of $\boldsymbol{M}_{\mathrm{f}}$ is much more probable than the change of $M_{\mathrm{f}}$ orientation on the exciton spin direction.

We used methods of polarized photoluminescence, namely the optical orientation [30], in order to get a detailed information about spin relaxation of excitons in the $48 \AA$ period $\mathrm{Cd}_{0.83} \mathrm{Mn}_{0.17} \mathrm{Te} / \mathrm{Cd}_{0.54} \mathrm{Mg}_{0.46} \mathrm{Te}$ superlattice. In this method excitons are created by a circularly polarized light, which determines the initial orientation of exciton spins. A degree of polarization of the PL signal $\left(P_{\mathrm{oo}}\right)$ is analyzed and it allows to find the ratio between lifetime and spin relaxation time of excitons. It was found that under nonselective excitation $P_{\mathrm{oo}} \approx 0$, this means that the free excitons lose their initial spin orientation very effectively due to the strong exchange interaction with the Mn spins [29,31]. However, the significant degree of optical orientation $P_{\mathrm{oo}}=0.27$ appears under selective excitation in the tail of localized exciton states.

Figure 5a shows two MP lines excited selectively with $\sigma^{+}$-polarized light $\left(E^{+}\right)$and detected in $\sigma^{+}\left(E^{+} A^{+}\right)$and $\sigma^{-}\left(E^{+} A^{-}\right)$polarizations. A difference in the intensities of the PL lines determines $P_{\mathrm{oo}}$. We note that the energy shift of the $E^{+} A^{-}$-line from the excitation energy is $\Delta f=3 \mathrm{meV}$ larger than the shift of the $E^{+} A^{+}$-line.

In Fig. $5 \mathrm{~b}$ the optical orientation degree is displayed by closed circles as a function of magnetic field. In nonmagnetic semiconductors $P_{\text {oo }}$ increases with magnetic fields applied in the Faraday geometry because of the suppression of spin relaxation mechanisms [30]. Contrary to that, in the semimagnetic superlattice we find an effective suppression of the degree of optical orientation at fields as small as $0.5 \mathrm{~T}$. Therefore, the nature of the optical orientation signal in the semimagnetic semiconductors differs from that in the nonmagnetic semiconductors. Warnock et al. suggested a model of optical orientation of excitons in the bulk semimagnetic 

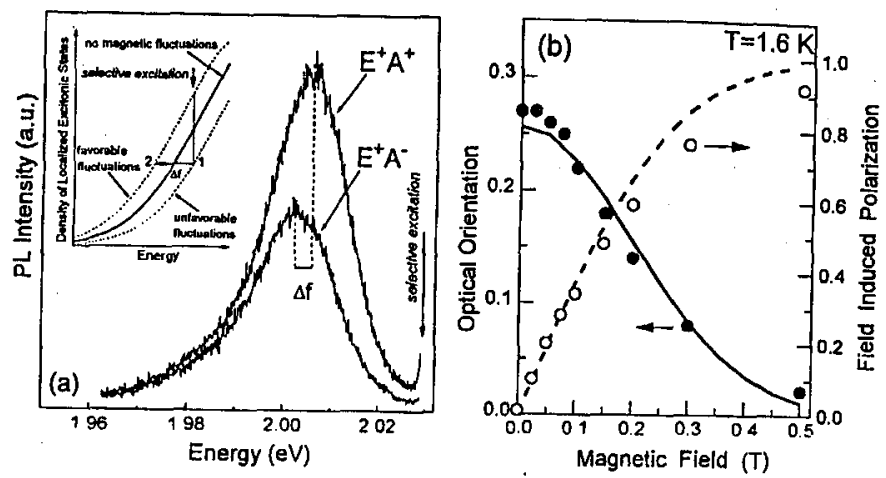

Fig. 5. (a) Photoluminescence spectra of a $48 \AA$ period $\mathrm{Cd}_{0.83} \mathrm{Mn}_{0.17} \mathrm{Te} /$ $\mathrm{Cd}_{0.54} \mathrm{Mg}_{0.46} \mathrm{Te} \mathrm{SL}$ excited selectively with $\sigma^{+}$-polarized light $\left(E^{+}\right)$and detected in $\sigma^{+}\left(E^{+} A^{+}\right)$and $\sigma^{-}\left(E^{+} A^{-}\right)$polarizations. The inset represents schematically a model of optical orientation in the semimagnetic semiconductors. (b) Degree of optical orientation and field-induced polarization as a function of magnetic field (Faraday geometry). Lines show the results of a model calculation.

semiconductors which accounts for Gaussian-distributed thermodynamical magnetic fluctuations inside the volume of exciton localization [28]. We modify this model for the 2D case considering that in SLs the heavy-hole exciton spin has only two opposite orientations parallel to the structure growth axis. We assume that after photogeneration selectively excited excitons first align their spins collinear with the orientation of $M_{\mathrm{f}}$ projection on the $z$-axis, and then form magnetic polarons, keeping their spin orientation constant. In this case $P_{o o}$ is determined by a difference in the probabilities to excite excitons at the magnetic fluctuations with different orientations. A scheme in the inset of Fig. 5 displays the situation where an exciton is excited at localized states with magnetic fluctuations. If the exciton is created in a "favorable" magnetic fluctuation (exciton spin parallel to the magnetic moment of fluctuation), which provides a minimum of the exciton energy, then it forms a MP with spin orientation coinciding with the polarization of the exciting photon (see $E^{+} A^{+}$-line). If the exciton is created in an "unfavorable" magnetic fluctuation, of which the magnetic moment is antiparallel to the exciton spin, then it first makes a spin-flip (as shown by arrow $1 \rightarrow 2$ ) and then forms a MP. Such MPs have an orientation antiparallel to the initial exciton orientation and contribute to the $\sigma^{-}$-detected PL (see $E^{+} A^{-}$-line). The energy shift of the polaron line in this case exceeds the MP energy by about two times the energy of the magnetic fluctuation. From our experiments we estimated the characteristic energy of the thermodynamical magnetic fluctuations affecting the localized excitons in the $\mathrm{Cd}_{0.83} \mathrm{Mn}_{0.17} \mathrm{Te} / \mathrm{Cd}_{0.54} \mathrm{Mg}_{0.46} \mathrm{Te}$ superlattice to be about $\Delta f / 2=1.5 \mathrm{meV}$. It is useful to note here that in structures with high Mn concentrations $(x>0.25)$ the "frozen" magnetic fields of Mn spin clusters can modify the spectrum of magnetic fluctuations and contribute to the optical orientation signal $[7,32]$.

The solid line in Fig. $5 \mathrm{~b}$ represents a result of the model calculation which was obtained without fitting parameters, as all required parameters were determined 
experimentally $[29,33]$. An excellent agreement between calculated and measured values of $P_{\text {oo }}$ justifies the assumption used in the model. Open circles in Fig. 5b show the magnetic-field-induced polarization of luminescence. Nonselective excitation was used in this experiment. The luminescence is unpolarized at zero field and the polarization degree gains its saturation level of 1.0 at a field of $0.5 \mathrm{~T}$. The values of the field-induced polarization degree are too small to be explained by the equilibrium thermal population of the exciton Zeeman sublevels, but can be well described in the frame of the model accounting thermodynamical magnetic fluctuations $[29,33]$. A result of the model calculation is shown by a dashed line.

It follows from the results presented above that the directional stabilization of the exciton spin (and respectively, $\boldsymbol{B}_{\text {ex }}$ ), which occurs in the process of the starting correlation between exciton and the system of $\mathrm{Mn}$ spins, is controlled by the thermodynamical fluctuations of magnetization. $\boldsymbol{B}_{\mathrm{ex}}$ is aligned along the magnetic moment of the magnetic fluctuation during time which is shorter than units of picoseconds, and then does not change its orientation during the process of the MP formation up to the exciton recombination.

In summary, the following hierarchy of relaxation times in the process of MP formation can be established on the base of reported results:

(i) The starting correlation between the exciton and the system of Mn spins is established during the time of order of one picosecond. It is determined by the relaxation of the exciton spin on the direction of fluctuation of magnetization.

(ii) The evolution of the MP energy covers the dynamical range from 50 up to $250 \mathrm{ps}$ and is controlled by the spin-spin interactions in the Mn spin system.

(iii) The directional relaxation time of the polaron magnetic moment exceeds the MP formation time at least by an order of magnitude [33]. It is considerably longer than the polaron lifetime ranged in QWs from $100 \mathrm{ps}$ up to $300 \mathrm{ps}$.

\section{Acknowledgments}

I appreciate fruitful collaboration with $\mathrm{K}$. Kavokin who contributed to the theoretical part of this paper as well as with I.A. Merkulov, G. Mackh, W. Ossau, A. Waag, G. Landwehr, R. Hellmann, E.O. Göbel, and A. Kavokin. I would like to thank V.P. Kochereshko, Yu.G. Semenov, C. Benoit à la Guillaume, T. Dietl, D. Scalbert, Yu.G. Kusraev, and A.V. Kudinov for helpful discussions. This work has been supported by the Deutsche Forschungsgemeinschaft through SFB 410.

\section{References}

[1] J.K. Furdyna, J. Appl. Phys. 64, R29 (1988).

[2] P.G. De Gennes, Phys. Rev. 118, 141 (1960).

[3] T. Kasuya, A. Yanase, Rev. Mod. Phys. 40, 684 (1968).

[4] P.A. Wolff, in: Semiconductors and Semimetals, Vol. 25, Eds. J.K. Furdyna, J. Kossut, Academic Press, London 1988, p. 413.

[5] A. Golnik, J. Ginter, J.A. Gaj, J. Phys. C 16, 6073 (1983),

[6] T. Itoh, E. Komatsu, J. Lumin. 38, 266 (1987).

[7] B.P. Zakharchenya, Yu.G. Kusraev, JETP Lett. 50, 225 (1989).

[8] V.F. Agekyan, A.Yu. Serov, Sov. Phys.-Solid State 32, 1951 (1990). 
[9] D.R. Yakovlev, W. Ossau, G. Landwehr, R.N. Bicknell-Tassius, A. Waag, I.N. Uraltsev, Solid State Commun. 76, 325 (1990).

[10] T. Dietl, P. Peyla, W. Grieshaber, Y. Merle d'Aubigné, Phys. Rev. Lett. 74, 474 (1995).

[11] M.R. Freeman, D.D. Awschalom, J.M. Hong, L.L. Chang, Phys. Rev. Lett. 64, 2430 (1990).

[12] D.R. Yakovlev, in: Festkörperprobleme/Advances in Solid State Physics, Vol. 32, Ed. U. Rössler, Vieweg, Braunschweig 1992, p. 251.

[13] D.D. Awschalom, M.R. Freeman, N. Samarth, H. Luo, J.K. Furdyna, Phys. Rev. Lett. 66, 1212 (1991).

[14] C. Benoit à la Guillaume, Phys. Status Solidi B 175, 369 (1993).

[15] A.V. Kavokin, K.V. Kavokin, Semicond. Sci. Technol. 8, 191 (1993).

[16] T. Kasuya, A. Yanase, T. Takeda, Solid State Commun. 8, 1543 (1970).

[17] S.M. Ryabchenko, Yu.G. Semenov, Sov. Phys.-Solid State 26, 2011 (1984).

[18] A.V. Kavokin, K.V. Kavokin, Sov. Phys.- Semicond. 25, 1053 (1991).

[19] C. Benoit à la Guillaume, Yu.G. Semenov, M. Combescot, Phys. Rev. B 51, 14124 (1995).

[20] G. Mackh, W. Ossau, D.R. Yakovlev, A. Waag, G. Landwehr, R. Hellmann, E.O. Göbel, Phys. Rev. B 49, 10248 (1994).

[21] D.R. Yakovlev, K.V. Kavokin, Comments on Condensed Matter Physics, 1996, in press.

[22] D.R. Yakovlev, W. Ossau, G. Landwehr, R.N. Bicknell-Tassius, A. Waag, S. Schmeusser, I.N. Uraltsev, Solid State Commun. 82, 29 (1992).

[23] D.R. Yakovlev, W. Ossau, G. Landwehr, R.N. Bicknell-Tassius, A. Waag, S. Schmeusser, I.N. Uraltsev, A. Pohlmann, E.O. Göbel, J. Cryst. Growth 117, 854 (1992).

[24] G. Mackh, M. Hilpert, D.R. Yakovlev, W. Ossau, H. Heinke, T. Litz, F. Fischer, A. Waag, G. Landwehr, R. Hellmann, E.O. Göbel, Phys. Rev. B 50, 14069 (1994).

[25] E.O. Göbel, R. Hellmann, G. Mackh, D.R. Yakovlev, W. Ossau, A. Waag, G. Landwehr, Mater. Sci. Forum 182-184, 735 (1995).

[26] J.J. Zayhowski, R.N. Kershaw, D. Ridgley, K. Dwight, A. Wold, R.R. Galazka, W. Giriat, Phys. Rev. B 35, 6950 (1987).

[27] R. Hellmann, A. Euteneuer, E.O. Göbel, G. Mackh, D.R. Yakovlev, A. Waag, G. Landwehr, J. Cryst. Growth 159, 976 (1996).

[28] J. Warnock, R.N. Kershaw, D. Ridgely, K. Dwight, A. Wold, R.R. Galazka, J. Lumin. 34, 25 (1985).

[29] V.P. Kochereshko, I.A. Merkulov, K.V. Kavokin, D.R. Yakovlev, G. Mackh, W. Ossau, A. Waag, G. Landwehr, in: Proc. Int. Conf. on Semiconductor Heteroepitaxy, Montpellier (France) 1995, Eds. B. Gil, R.L. Aulombard, World Scientific, Singapore 1995, p. 218.

[30] Optical Orientation, Eds. F. Meier, B.P.Zakharchenya, North-Holland, Amsterdam 1984. 
[31] V.P. Kochereshko, I.A. Merkulov, G.R. Pozina, I.N. Uraltsev, D.R. Yakovlev, W. Ossau, A. Waag, G. Landwehr, Solid-State Electron. 37, 1081 (1994).

[32] A.V. Kudinov, Yu.G. Kusraev, V.N. Yakimovich, Phys. Solid State 37, 359 (1995).

[33] I.A. Merkulov, D.R. Yakovlev, K.V. Kavokin, G. Mackh, W. Ossau, A. Waag, G. Landwehr, JETP Lett. 62, 335 (1995). 